\section{Pompe disease, a storage cardiomyopathy}

\section{Tiziana Felice \\ Cardiology Department, Mater Dei \\ Hospital, Msida, Malta}

\begin{abstract}
Pompe disease also known as glycogen storage disease type II, is a rare and progressive lysosomal storage disorder caused by the deficiency of the enzyme acid $\alpha$-glucosidase. This results in the accumulation of glycogen in various tissues particularly involving the heart, skeletal muscle and liver. It is inherited in an autosomal recessive manner due to mutations in the $G A A$ gene. There are several known pathogenic variants, some of which are particularly common in certain geographical regions. Pompe disease is a single disease exhibiting a heterogeneous clinical spectrum depending on the extent of enzyme deficiency, the age of onset, the progression of the disease and the degree of organ involvement. It may lead to muscle weakness, hypotonia, respiratory compromise and premature death. Pompe disease is classically divided into two forms, infantile and late-onset disease. The infantile form is further subdivided into classical and non-classical subtypes. Cardiac involvement is particularly seen in the infantile phenotype of the condition, presenting as severe cardiomyopathy associated with conduction abnormalities. Enzyme replacement therapy with recombinant human acid $\alpha$-glucosidase is the approved treatment option for patients with this metabolic condition. Further research is currently being done to explore more treatment options. One must keep in mind other metabolic and mitochondrial conditions, which may give a similar cardiac and neurological clinical picture.
\end{abstract}

\section{Introduction}

Pompe disease (PD) also known as glycogen storage disease type II (GSD II), is an inherited condition caused by the absence of the enzyme acid $\alpha$-glucosidase (GAA). This lysosomal enzyme is responsible for the degradation of glycogen into glucose. ${ }^{1} \mathrm{PD}$ is a rare systemic disease with an overall incidence of 1:40,000 births, but the incidence varies depending on ethnicity and geographical regions. ${ }^{2,3}$ It is inherited in an autosomal recessive manner and the genetic abnormality is located in the GAA gene, found on the long arm of chromosome 17..$^{2,3}$ The genetic mutations result in the accumulation of glycogen either within the lysosomes or free within the cytoplasm. ${ }^{4}$ The total absence of $\alpha$-glucosidase activity causes early onset of the disease with death in the first years of life from cardio-respiratory failure secondary to severe left ventricular hypertrophy $(\mathrm{LVH}) .^{5}$ On the other hand, a partial deficiency of this enzyme results in a milder, late-onset, slowly progressing sub-type of the condition. ${ }^{6,7}$ Cardiac involvement may be the initial and predominant manifestation of defects in human glycogen metabolism. ${ }^{5}$ The literature search was performed using the PubMed database search engine. The keywords used were Pompe disease and cardiomyopathy, Pompe disease and cardiac involvement, and glycogen storage disease type II cardiomyopathy.

\section{Pathology}

The enzyme deficiency in PD results in the accumulation of glycogen in various tissues. Lysosomal disruption occurs and excess autophagic vesicle build up is seen inside the myofibres causing progressive cardiac, motor and respiratory failure. The clinical outcome correlates with the degree of enzyme deficiency. Histochemistry in PD shows glycogen accumulation in membrane-bound vesicles known as lysosomes. These changes are widespread occurring in many organs and tissues; skeletal muscle often being the most studied tissue. ${ }^{8}$ Microscopically muscle fibre degeneration is characterised by the presence of shrunken nuclei with condensed chromatin. Haematoxylin and eosin staining help to show areas of vacuolization within the myocyte whilst periodic acid Schiff staining highlights the abundance of glycogen. The increased lysosomal abundance is demonstrated by acid phosphatase staining. ${ }^{9}$ The histological and ultrastructural changes described occur in both skeletal and cardiac muscle.

\section{Clinical classification}

PD may be classified into two subtypes: infantile and late onset forms.

\section{Infantile onset Pompe disease}

Infantile onset Pompe disease (IOPD) is the classic sub-type of the condition, which was first described in 1934 and is associated with severe hypertrophic cardiomyopathy in $88-100 \%$ of patients. ${ }^{10,11}$ Severe muta-
Correspondence: Tiziana Felice, Cardiology Department, Mater Dei Hospital, Msida, Malta.

Tel.: +35.679260381.

E-mail: tizianafelice2603@gmail.com

Key words: Pompe disease; glycogen storage disease type II; acid $\alpha$-glucosidase cardiomyopathy.

Conflict of interest: the author declares no potential conflict of interest.

Received for publication: 18 June 2017 Revision received: 11 September 2017. Accepted for publication: 21 September 2017.

This work is licensed under a Creative Commons Attribution NonCommercial 4.0 License (CC BY-NC 4.0).

CCopyright T. Felice, 2017

Licensee PAGEPress, Italy

Cardiogenetics 2017; 7:6857

doi:10.4081/cardiogenetics.2017.6857

tions in the GAA gene cause complete or near-complete enzyme deficiency resulting in a severe clinical phenotype characterised by cardiac involvement, respiratory distress, feeding problems, macroglossia, motor delay, failure to thrive, muscle weakness, hypotonia (floppy baby) and organomegaly. ${ }^{12}$ Symptoms usually commence at approximately 1.6 months if untreated, causing cardiomegaly (92-96\%) and congestive heart failure (50\%), besides respiratory distress (78\%), muscle weakness (63-96\%), and feeding difficulties $(57 \%) .{ }^{11,12}$ Without treatment, the disease progresses rapidly with $92 \%$ of patients reported to die before reaching 1 year of age secondary to respiratory insufficiency. ${ }^{11,12}$ Individuals affected by IOPD may be apparent in utero but more typically the clinical onset is during the first few months of life..$^{13}$ There is a less severe form of IOPD (nonclassic IOPD) which still presents in the first year of life but it exhibits a slower clinical progression and the cardiac involvement is less severe. Affected patients still develop peripheral muscle weakness, cardiomyopathy and occasionally macroglossia and organomegaly, but in milder forms. ${ }^{1}$

\section{Late onset Pompe disease}

Late-onset Pompe disease (LOPD) includes a childhood, juvenile or muscular variant of the condition. It is a heterogeneous group usually presenting after one year of age and typically does not including severe cardiac involvement. LOPD also encompasses the adult-onset form of the condition, which presents with a slow pro- 
gressive myopathy predominantly involving the skeletal muscle. The onset of this form of PD may occur as late as the second and sixth decades of life.

\section{Cardiovascular involvement}

\section{Myocardial involvement}

$\mathrm{PD}$ is one of the commonest causes of familial (idiopathic) hypertrophic cardiomyopathy in neonatal and paediatric age. ${ }^{14}$ Glycogen accumulates within the cardiac myocytes causing a severe form of hypertrophic cardiomyopathy with significant asymmetrical thickening of the septum. In infants affected by the condition, severe concentric hypertrophy is seen also affecting the septum and the free walls of both the right and the left ventricles resulting in biventricular hypertrophy (Figure 1). In $30 \%$ of cases, systolic anterior motion of the anterior mitral valve leaflet is present which may contribute to a worse prognosis. ${ }^{15,16}$ Diastolic and systolic dysfunction may also be observed. The dysfunctional left ventricle may become dilated as well as hypertrophied, and there can be underlying myocardial fibrosis detected by cardiac magnetic resonance imaging. ${ }^{17}$ In a study of 40 patients with IOPD, the development of systolic dysfunction, defined as a left ventricular ejection fraction of less than $40 \%$, developed after 5 months of age. ${ }^{18}$

In non-classic IOPD, cardiac involvement as conduction abnormalities and electrocardiogram (ECG) signs of ventricular hypertrophy are generally part of the disease spectrum. On echocardiography, cardiac hypertrophy is less severe and progressive and lacks the left ventricular obstruction and systolic dysfunction that significantly worsens the classic phenotype. Suzuki et al. have reported that the clinical spectrum in this form of PD may be varied. ${ }^{19}$

\section{Conduction system involvement}

In PD, glycogen not only accumulates in the heart muscle but also in the conducting tissue of the heart, explaining the classi- cal electrocardiographic abnormalities observed in this systemic condition (Figure 2). Electrocardiographic features of PD include a short PR interval, delta waves, pre-excitation patterns particularly of Wolff-Parkinson-White (WPW) morphology, left axis deviation, left ventricular hypertrophy by voltage criteria with repolarisation changes, atrio-ventricular blocks, and bundle branch block abnormalities. In this disease, the pre-excitation pattern on the ECG does not signify the presence of an accessory pathway. It is thought that this ECG pattern occurs either due to the direct insulating affect of glycogen on the conducting system or by the anatomical interruption of the annulus fibrosus, giving rise to an indirect insulating effect between the atria and the ventricles. ${ }^{16,20}$

\section{Arrhythmias}

Different types of cardiac arrhythmias have been observed in patients with PD. The arrhythmias described are common unspecified sinus arrhythmias, supraventricular tachycardia (SVT), sick sinus syndrome, Lown IVb ventricular arrhythmia and WPW syndrome. ${ }^{21-23}$ Furthermore, WPW is also associated with supraventricular tachycardia. One study reported that several patients suffering from PD with WPW syndrome, experienced recurrent SVT

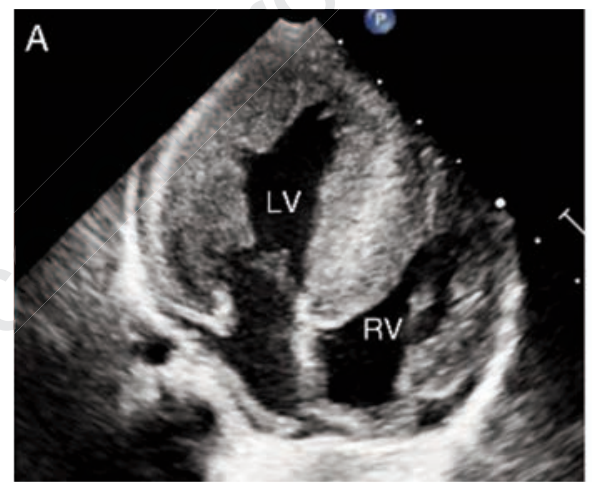

episodes requiring hospital admission. ${ }^{21}$ In a French registry, complete heart block was observed in approximately $3 \%$ of LOPD patients. ${ }^{24}$ Supraventricular and ventricular tachyarrhythmias are also relatively common affecting up to $29 \%$ of patients with LOPD. ${ }^{23}$ The electrocardiogram of some patients with PD may suggest ventricular pre-excitation, but pathology studies of mouse models show generalized disruption of the annulus fibrosis associated with glycogen storage disease rather than the presence of a myocardial bypass tract. ${ }^{14,17}$ An insulating effect on the cardiac conduction system by the glycogen within the myocytes, disrupts the normal conduction barriers between the atria and the ventricles thus enhancing electrical conduction. This phenomenon has implications in the treatment of supraventricular tachycardia due to its poor amenability to radiofrequency catheter ablation. ${ }^{14}$

\section{Cardiovascular involvement in IOPD versus LOPD}

Cardiac involvement in PD was previously recognized only in infantile cases, but cardiac hypertrophy, heart rhythm disturbances and aortic abnormalities are now also recognized in LOPD (Table 1). In infantile PD, the deficit of lysosomal GAA is often complete. Accordingly, in IOPD the

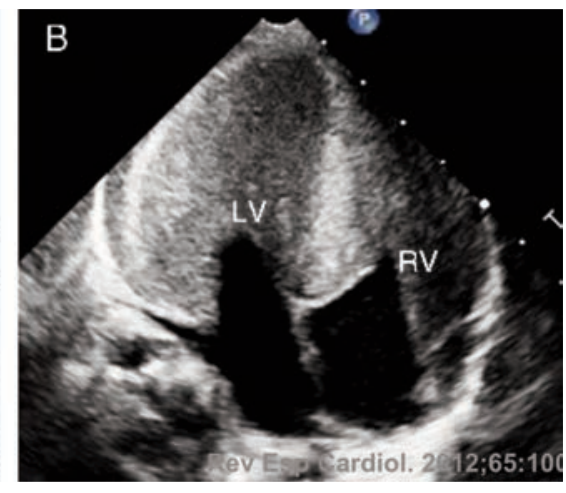

Figure 1. A) Hypertrophic cardiomyopathy in a patient with IOPD, during diastole. B) Hypertrophic cardiomyopathy in a patient with IOPD during systole. Cavity obliteration of the left ventricle is seen. [Reproduced with permission from: Bonilla-Palomas JL, et al. When should we start enzyme replacement therapy for infantile Pompe disease with severe cardiomyopathy? Rev Esp Cardiol 2012;65:100-2. Copyright (C) 2012 Sociedad Española de Cardiología. Published by Elsevier España, S.L.U. All rights reserved.]

Table 1. The various features of cardiac involvement in IOPD and LOPD.

\begin{tabular}{|c|c|c|c|}
\hline & Cardiovascular findings & IOPD & LOPD \\
\hline ECG & $\begin{array}{l}\text { Short PR interval, delta waves, pre-excitation patterns, WPW morphology, left axis deviation, } \\
\text { left ventricular hypertrophy by voltage criteria with repolarization changes, atrioventricular } \\
\text { conduction and bundle branch block abnormalities }\end{array}$ & $* * *$ & $* *$ \\
\hline Echocardiogram & $\begin{array}{l}\text { Biventricular hypertrophy, asymmetrical septal hypertrophy, systolic anterior motion of the anterior } \\
\text { mitral valve leaflet, left ventricular outflow tract obstruction, systolic and diastolic dysfunction }\end{array}$ & $* * *$ & $*$ \\
\hline Arrhythmias & Sinus arrhythmias, supraventricular tachycardia, sick sinus syndrome, Lown IVb ventricular arrhythmia, WPW & $* * *$ & $* *$ \\
\hline Vascular & Dilated aortopathy, aneurysm formation and dissection & * & $* * *$ \\
\hline
\end{tabular}

*Shows the increased frequency and severity in both categories. 
storage of glycogen in the heart is of considerable magnitude with consequent increase in mass and functional alterations of the heart. In contrast in LOPD, there is partial GAA enzyme deficiency $(2-40 \%$ of normal activity) and consequently structural and functional cardiac alterations are absent or mild, despite severe musculoskeletal changes. The metabolism and storage of glycogen is also different in the two groups, with less glycogen accumulation in cardiac muscle in the LOPD subgroup of patients. ${ }^{25,26}$ The residual activity of the GAA enzyme would be just sufficient to eliminate the accumulation of glycogen in cardiac but not in skeletal muscle. ${ }^{27-29}$

Controversy exists on the prevalence of cardiac involvement in LOPD patients. Soliman and colleagues assessed 46 patients with LOPD and found that there were no major cardiac abnormalities. The cardiac pathology seen in this group included isolated low systolic mitral annular velocities and mild left ventricular diastolic dysfunction, which could have been explained by a history of hypertension and/or advanced age rather than by LOPD. ${ }^{22}$ Forsha et al., report limited cardiac involvement in 87 patients with LOPD. These changes included left ventricular hypertrophy, short PR interval on ECG, and a decreased ejection fraction on echocardiogram. ${ }^{21}$ Morris and colleagues, on the other hand, demonstrated that individuals with LOPD, had no myocardial features of the disease when compared to healthy subjects. ${ }^{30}$ A recent study was performed analyzing comprehensively the heart with modern and sensitive myocardial techniques. No evidence of structural or functional alterations in adults with LOPD was seen. Both structural and functional cardiac features were normal in these patients. ${ }^{13}$

Gungor et al. described the survival of 268 patients with LOPD, collected in a prospective international observational study conducted between 2002 and 2009. Out of 34 deaths reported, the cause of death was recognized in 9 patients, including 1 patient who died of aortic dissection. ${ }^{31}$ In a German study of LOPD patients, dilated aortopathy, aneurysm formation and dissection, was seen in up to $12.5 \%$ of patients. ${ }^{22}$ Smooth muscle involvement occurs in LOPD patients as shown by the presence of increased aortic stiffness, secondary to glycogen accumulation in the vessel wall, reducing vascular elasticity. ${ }^{32}$ Severe vacuolization of vascular smooth muscle with accumulation of glycogen, particularly involving large and small cerebral arteries with aneurysm formation have previously been reported, reinforcing the need of cardiovascular follow-up in this cohort of patients..$^{33,34}$

The latest guidelines on PD highlighted that cardiac involvement in adult PD remains inconclusive. ${ }^{29}$ Although the current evidence suggests that there is minimal cardiac involvement in LOPD, there are reported cases of individuals with LOPD and cardiomyopathy alone. ${ }^{35,36}$ Therefore cardiomyopathy and arrhythmias should be recognized as possible LOPD clinical features, although not as common as in IOPD. Clinical screening for patients with LOPD, by performing yearly ECGs, echocardiograms and ambulatory ECG monitoring is highly recommended. Pathological changes in skeletal muscle, on the other hand, are prominent throughout the entire spectrum. ${ }^{6,7}$

\section{Differential diagnoses}

Hypertrophic cardiomyopathy is an umbrella term, which encompasses various conditions. The commonest form of hypertrophic cardiomyopathy is the sarcomeric type, secondary to mutations occurring in the genes encoding for sarcomeric proteins. A similar cardiac phenotype is seen in a number of conditions including syndromic, mitochondrial and metabolic pathologies. ${ }^{37}$ Ventricular pre-excitation or tall $\mathrm{R}$ waves on the ECG and the presence of idiopathic left ventricular hypertrophy in children are a common feature of storage diseases (AMPkinase disease, Danon disease), and mitochondrial disorders (MELAS, MERFF). Danon disease is an X-linked dominant glycogen storage disorder due to the absence of the lysosomal-associated membrane protein 2 (LAMP-2) which may be diagnosed by immunohistochemistry or by genetic sequencing. Compared with PD, cognitive impairment and retinitis pigmentosa may be distinctive features of this condition. ${ }^{17}$ AMPkinase disease may result in a similar phenotype but is inherited in an autosomal dominant manner.

Mitochondrial disorders may cause left ventricular hypertrophy and are associated with a number of extra cardiac features. Mitochondrial myopathy, encephalopathy, lactic acidosis and stroke are seen in MELAS syndrome. Myoclonic epilepsy with ragged red fibres is a multisystem disorder causing generalised seizures, ataxia and later on dementia. Optic atrophy and hearing loss may be present. A family pedigree indicating maternal inheritance may point to the diagnosis.

\section{Investigations}

The diagnosis of PD is established by demonstrating a deficiency in acid $\alpha$-glucosidase enzyme activity, combined with finding disease-causing mutations using DNA analy- sis of the GAA gene. Rapid and sensitive analysis of the GAA enzyme activity can be performed on dried blood spots when using standard conditions. Dried blood spot enzyme analysis is convenient as well as time- and cost-effective, but may give falsepositive results and thus requires confirmatory DNA testing to make a diagnosis of PD. ${ }^{38}$

DNA analysis may be performed using traditional targeted Sanger sequencing, once the enzyme deficiency is obtained. Now newer methods of DNA analysis are available involving, massive parallel sequencing which may be used as the initial test for analysis of the GAA gene. PD is inherited in an autosomal recessive manner. Hence if a patient is homozygous for the pathogenic mutations (present in both alleles), the diagnosis is confirmed. On the other hand, the presence of one known mutation and a sequence variant (with unproven pathogenicity), requires confirmation using an enzymatic assay from such sources as a dried blood spot, a skin fibroblast culture, a peripheral blood lymphocyte culture, or histological evidence from a muscle biopsy. ${ }^{38}$

A number of biomarkers may be elevated in this condition. Serum creatinine kinase concentration is elevated (as high as $2000 \mathrm{IU} / \mathrm{L}$; normal range: 60-305 IU/L) in all individuals with IOPD and in some subjects with LOPD. ${ }^{27}$ Another biomarker which may be elevated in PD is glucose tetrasaccharide, which is a tetramer identified in the urine and recently applied in GSD type II. It is a biomarker associated with skeletal muscle glycogen content, and raised levels are found in $94 \%$ of patients with PD. It is not specific for this condition as it may rise in other inherited neuromuscular diseases, trauma, acute pancreatitis and certain malignancies. ${ }^{39}$

\section{Recommended cardiac investiga- tions}

In the infantile form, a chest X-ray will convey gross cardiomegaly, which may be done during the first evaluation (Figure 3). The assessment of cardiac function by echocardiography is an essential part of the ongoing management of Pompe patients. Hypertrophy, predominantly affecting the left ventricular posterior wall and the interventricular septum is typically seen in infants at diagnosis and may also be identified prenatally. ${ }^{13}$ Left ventricular outflow tract obstruction may be present. The hypertrophic cardiomyopathy results in systolic and diastolic dysfunction that may progress to congestive heart failure. Dilatation of the 
left ventricle with a low ejection fraction may develop. Initial findings of increased wall thickness and left ventricular (LV) mass need to be evaluated on a regular basis by a standardized approach since establishing the stage of the cardiomyopathy is useful in determining treatment. This imaging modality may help with following disease progression and response to intervention. The LV mass should be evaluated by two dimensional measurements using the length-area relationship. ${ }^{27}$ The absolute mass value (not indexed for BSA) is compared to a normal control population to yield a $\mathrm{z}$-score which is an indicator of how far the patient exceeds the mean left ventricular mass in normal subjects. This is particularly useful in the paediatric population. Functional measurements including 2-D ejection fraction and the myocardial performance index are helpful in longitudinal follow-up. In IOPD echocardiography should be performed at baseline then at 6 monthly intervals. Once enzyme replacement therapy is commenced closer echocardiographic monitoring is required as the ejection fraction may deteriorate in the first 12-24 weeks of therapy. In LOPD patients, yearly echocardiography is recommended. ${ }^{27}$

Cardiac magnetic resonance imaging may be recommended to assess cardiac morphology, enhanced with gadolinium analysis for cardiac tissue characterization. Ambulatory ECG monitoring is recommended to assess for cardiac arrhythmias, at least 6 monthly in IOPD and yearly in LOPD patients.

\section{Genetics}

PD is inherited in an autosomal recessive manner due to mutations of the GAA gene located on the long arm of chromosome 17. Biallelic GAA pathogenic variants may produce essentially no enzyme activity, resulting in IOPD. ${ }^{40}$ Several combinations of other pathogenic variants result in some residual enzyme activity, causing milder forms of this disease. The age of onset and progression of $\mathrm{PD}$, are most likely directly proportional to the residual GAA activity.

Genotype-phenotype correlations have been made depending on the type of GAA pathogenic variant present. Variants that cause mRNA instability such as nonsense mutations are more commonly seen in IOPD since they result in near complete absence of GAA enzyme activity. On the other hand, GAA missense and splicing site pathogenic variants may result in either complete or partial absence of GAA enzyme activity and therefore may cause both IOPD and LOPD. ${ }^{41}$

Several genotype-phenotype correlations have been observed with other specific pathogenic variants, sometimes endemic in certain populations. The pathological variant p.Glu76ArgfsTer45 (c.525delT) is especially common among the Dutch population. ${ }^{41}$ It results in negligible GAA enzyme activity and is classified with the more severe alterations. Deletion of exon 18 (p.Gly828_Asn882del;c.2482_2646del) is also a common pathogenic variant again

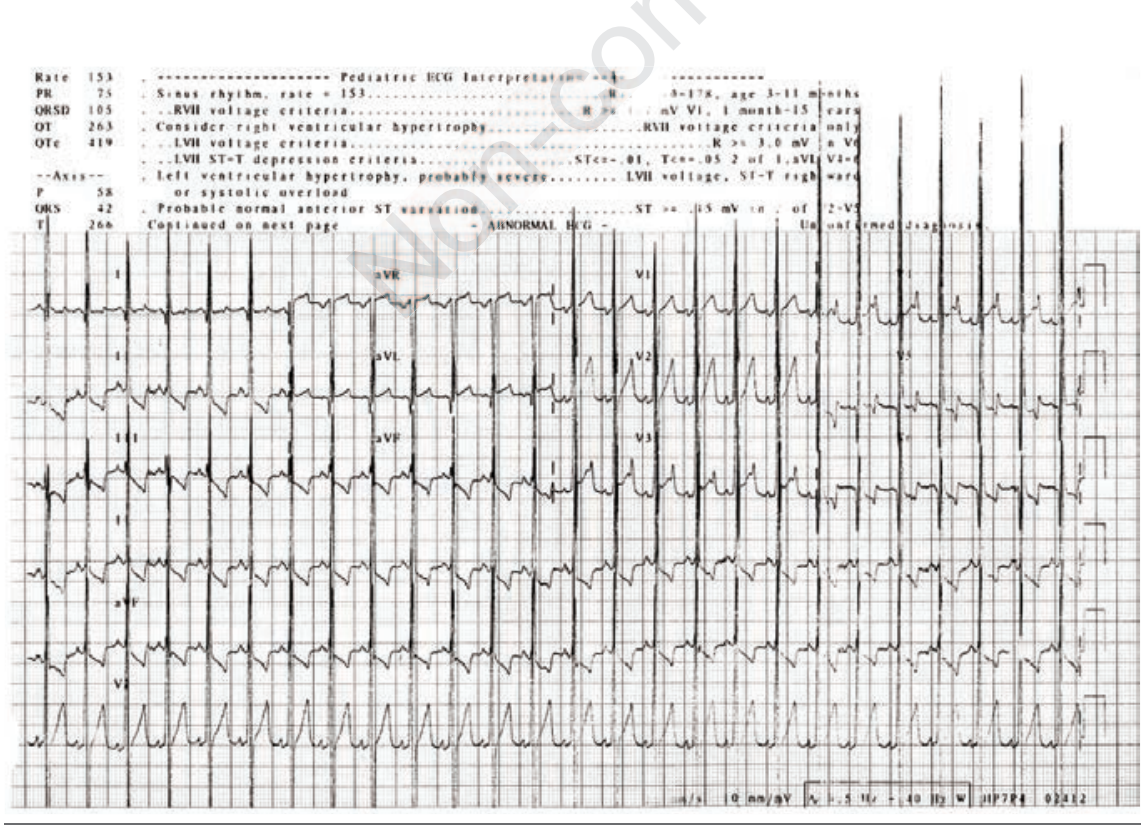

Figure 2. Electrocardiogram in a patient with Pompe disease. Prominent $\mathrm{R}$ waves, features of left ventricular hypertrophy by voltage criteria with repolarization abnormalities are seen. [Reproduced with permission from: Poon GWK, et al. Variable response to enzyme replacement therapy in two Chinese children with infantile-onset Pompe disease in Hong Kong. HK J Paediatr (New Series) 2009;14:243-51. Medcom Ltd.]

particularly among the Dutch. It results in negligible GAA enzyme activity and is considered as one of the more severe diseasecausing variants. ${ }^{42}$ The mutation c.336$13 \mathrm{~T}>\mathrm{G}$ is seen in $36 \%$ to $90 \%$ of late onset GSD II and is not associated with IOPD. ${ }^{43}$ This pathogenic mutation leads to a leaky splice site resulting in greatly diminished, but not absent, GAA enzyme activity. Another pathogenic variant p.Asp645Glu, is seen in a high proportion ( $80 \%$ ) of IOPD patients in Taiwan and China and is associated with a haplotype suggesting a founder effect. $^{44}$ Another disease-causing variant p.Arg854Ter, is frequently associated with IOPD. This pathogenic variant has been observed in up to $60 \%$ of individuals of African descent who had a common haplotype, suggesting a founder effect. ${ }^{45}$

\section{Management of Pompe disease cardiomyopathy}

Treatment of Pompe cardiomyopathy is directed by the patients' symptoms. A consultation with a cardiologist is required to outline the treatment and management of the affected patient. PD patients with severe ventricular hypertrophy and left ventricular outflow tract obstruction are managed with $\beta$-blockade and careful monitoring of fluid status. Regular assessment of atrio-ventricular conduction is required with ECG and $24 \mathrm{~h}$ ambulatory ECG monitoring, to prevent complete heart block. If this occurs, permanent pacing may be required. Avoidance of dehydration or hypotension is important, as this may precipitate ventricular arrhythmia and cardiovascular collapse.

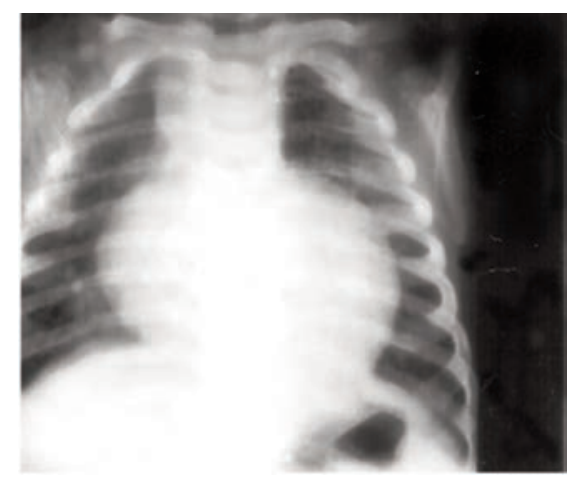

Figure 3. Significant cardiomegaly on chest $\mathrm{x}$-ray in a patient with IOPD with cardiac involvement. [Reproduced with permission from: Amalfitano A, et al. Recombinant human acid [agr]-glucosidase enzyme therapy for infantile glycogen storage disease type II: Results of a phase I/II clinical trial. Genet Med 2001;3:132-8. Nature Publishing Group.] 
Inotropic medications and afterload reduction may worsen outflow tract obstruction and should only be used in the setting of congestive heart failure. ${ }^{38}$ Supraventricular tachyarrhythmias may be controlled with $\beta$ blockade. Careful monitoring for ventricular ectopy is warranted both prior to the initiation of enzyme replacement therapy (ERT) and during treatment. ${ }^{19}$ The presence of cardiomyopathy in older children with PD may not necessarily indicate a poor prognosis unlike in classic IOPD. ${ }^{7}$ Cardiomyopathy cannot be used as a single criterion for the classification of $\mathrm{PD}$ in infants and children. Cardiomegaly needs to be monitored even after 1 year of age. ${ }^{46}$

\section{Enzyme replacement therapy}

Enzyme replacement therapy (ERT) has been found to reverse the cardiomyopathy, improve the clinical course and the expected outcome of PD patients. ${ }^{31,46}$ Significant regression of left ventricular hypertrophy occurs as early as two months after initiation of ERT but may not resolve completely in infants started on treatment after five months of age, or in those subjects with a marked increase in the left ventricular mass index. ${ }^{19}$ The normalization of left ventricular mass index has been shown to persist in long-term survivors on ERT. ${ }^{47}$ In the first two months of treatment a transient decrease in left ventricular ejection fraction is often noted, which typically returns to baseline by six months of therapy. ${ }^{48}$ Similarly the ECG changes associated with PD such as the short PR interval, and elevated QRS voltages, resolve in patients undergoing ERT. ${ }^{48}$ In a study by Chen et al. four patients with left ventricular outflow tract obstruction on beta-blockers were noted to have no gradient across the left ventricular outflow tract following one month of ERT. ${ }^{48}$ Despite the improvement in cardiac hypertrophy, PD patients undergoing ERT still have an increased risk of long-term conduction abnormalities and tachyarrhythmias. ${ }^{19}$

In general, the monitoring of patients with PD requires a coordinated multi-disciplinary approach by a specialist and individuals who have expertise in the care of these patients. ERT is the only established drug therapy for patients with PD as it targets the enzyme deficiency. Recombinant human acid $\alpha$-glucosidase (RhGAA) is the only approved ERT currently available and is administered as an intravenous infusion every two weeks. The bioavailability of the recombinant enzyme may be influenced by the development of anti-GAA neutralizing antibodies, which occur at the start of treat- ment. In cases of IOPD, the probability of developing high titres of anti-GAA antibodies is primarily affected by their cross-reactive immunologic material (CRIM) status. ${ }^{38}$

Patients with IOPD should be offered ERT at a dose of $20 \mathrm{mg} / \mathrm{kg}$ every other week. As regards to LOPD, a randomized, placebo-controlled prospective trial of rhGAA was performed in which 90 patients aged $\geq 8$ years of age were randomly assigned to receive treatment either with rhGAA or placebo. ${ }^{49}$ Subjects on the active treatment showed significant improvement in the forced vital capacity (FVC) and the six minute walk test. ${ }^{49}$

Although ERT should be initiated as soon as the diagnosis of IOPD or symptomatic PD is established, it may be appropriate to determine the patients' CRIM status prior to initiating treatment, as individuals who do not produce CRIM, may develop higher anti-rhGAA antibodies during ERT and may require modified therapy protocols using immunomodulation, ideally prior to the first infusion. ${ }^{50} \mathrm{New}$ therapeutic approaches are being tested, one being the development of an antibody-enzyme protein infusion aimed to target both the glycogen within the lysosomes but also within the cytoplasm. ${ }^{51}$

\section{Conclusions}

PD is a complex metabolic disease with a heterogeneous clinical spectrum. The severity is related to the varying levels of residual enzyme activity as dictated by the individual's genetic background. The PD cardiomyopathy is often seen in the infantile form of this condition, but cardiac involvement may still be present but less aggressive, in the late-onset sub-type. A multidisciplinary approach is required for the holistic care and management of PD patients. Regular cardiac consultation together with ECG, echocardiography and 24-hour arrhythmia monitoring is necessary. Early commencement of ERT is recommended. Considering the overall limitations of ERT with its high costs, innovative therapeutic approaches are now under development, to reduce the development of anti-rhGAA antibodies as well as to target both lysosomal and cytoplasmic glycogen.

\section{References}

1. Hers HG. Alpha-glucosidase deficiency in generalized glycogen storage disease (Pompe's disease). Biochem J 1963;86: 11-6.
2. Ausems MG, Verbiest J, Hermans MP, et al. Frequency of glycogen storage disease type II in the Netherlands: implications for diagnosis and genetic counseling. Eur J Hum Genet 1999;7: 713-6.

3. Martiniuk F, Chen A, Mack A, et al. Carrier Frequency for glycogen storage disease type II in New York and estimates of affected individuals born with the disease. Am J Med Genet 1998;79: 69-72.

4. Raben N, Plotz P, Byrne BJ. Acid alphaglucosidase deficiency (glycogenesis type II, Pompe disease). Curr Mol Med 2002;2:145-66.

5. Arad M, Maron BJ, Gorham JM, et al. Glycogen storage diseases presenting as hypertrophic cardiomyopathy. N Engl J Med 2005;352:362-72.

6. Hagemans ML, Winkel LP, Hop WC, et al. Disease severity in children and adults with Pompe disease related to age and disease duration. Neurology 2005;64:2139-41.

7. Winkel LP, Hagemans ML, Van Doom PA, et al. The natural course of nonclassic Pompe disease; a review of 225 published cases. J Neurol 2005;252: 875-84.

8. Dobowtiz V, Sewry CA, Oldfors A. Muscle biopsy: a practical approach. $3^{\text {rd }}$ ed. Amsterdam: Elsevier Saunders: 2007.

9. Werneck LC, Lorenzoni PJ, Kay CS, et al. Muscle biopsy in Pompe disease. Arq Neuro-psiquiat 2013;71:284-9.

10. Pompe JC. Over idiopatische hypertrophie van het hart. Ned Tijdshr Geneeskd 1932;67:304.

11. Van Den Hout HM, Hop W, Van Diggelen OP, et al. The natural course of infantile Pompe's disease: 20 original cases compared with 133 cases from the literature. Pediatrics 2003;112:332-40.

12. Case LE, Bjartmar C, Morgan C, et al. Safety and efficacy of alternative alglucosidase alfa regimens in Pompe disease. Neuromuscul Disord 2015;25: 231-22.

13. Handan MA, El-Zoabi BA, Begam MA, et al. Antenatal diagnosis of pompe disease by foetal echocardiography: impact on outcome after the initiation of enzyme replacement therapy. J Inherit Metab Dis 2010;33:333-9.

14. Elliott P, Andersson B, Arbustini E, et al. Classification of the cardiomyopathies: a position statement from the European Society of Cardiology Working Group on Myocardial and Pericardial Diseases. Eur Heart J 2008;29:270-6.

15. Limongelli G, Fratta F. Cardiovascular 
involvement in Pompe disease. Proceedings of the meeting course on type II glycogenosis: a network for the treatment of Pompe disease. Acta Myol 2011;30:202-3

16. Parenti G, Di Iorio G, Sampaolo S, et al. Molecular basis and clinical management of Pompe disease. Special Issue on lysosomal disease. Cardiogenetics 2013;3(s1):e5.

17. Barker PC, Pasquali SK, Darty S, et al. Use of cardiac magnetic resonance imaging to evaluate cardiac structure, function and fibrosis in children with infantile Pompe disease on enzyme replacement therapy. Mol Genet Metab 2010;101:332-7.

18. Chen LR, Chen CA, Chiu SN, et al. Reversal of cardiac dysfunction after enzyme replacement in patients with infantile-onset Pompe disease. J Paediatr 2009;155:271-5.

19. Suzuki Y, Tsuiji A, Omura K, et al. Km mutant of acid alpha-glucosidase in a case of cardiomyopathy without signs of skeletal muscle involvement. Clin Ven 1988;33:376-85.

20. Arad M, Moskowitz IP, Patel VV, et al. Transgenic mice overexpressing mutant PRKAG2 define the cause of WolffParkinson-White syndrome in glycogen storage cardiomyopathy. Circulation 2003;107:2850-6.

21. Forsha D, Li JS, Smith PB, et al. Late onset treatment study investigators. Cardiovascular abnormalities in late onset Pompe disease and response to enzyme replacement therapy. Genet Med 2011;13:625-31.

22. Soliman OI, van der Beek NA, van Doorn PA, et al. Cardiac involvement in adults with Pompe disease. J Intern Med 2008;4:333-9.

23. Schuller A, Wenninger S, Strigl-Pill N, Schoser B. Towards deconstructing the phenotype of late-onset Pompe disease. AM J Med Genet Sem Med Gen 2012;160C:80-8

24. Sacconi S, Wahbi K, Theodore G, et al. Atrioventricular block requiring pacemaker in patients with late-onset Pompe disease. Neuromuscul Disord 2014;24:648-50.

25. Reuser A, Kroos MA, Hermans MM. Glycogenosis type II (acid maltase deficiency). Muscle Nerve 1995;3:61-9.

26. Merin RG. Myocardial metabolism for the toxicologist. Environ Health Perspec 1978;26:169-74.

27. Kishnani PS, Steiner RD, Bali D, et al. Pompe disease diagnosis and management guideline. Genet Med 2006;8:267-
88.

28. Fukuda T, Roberts A, Ahearn M, et al. Autophagy and lysosomes in Pompe disease. Autophagy 2006;2:318-20.

29. Cupler EJ, Berger KI, Leshner RT, et al. AANEM consensus committee on lateonset Pompe disease. Consensus treatment recommendations for late-onset Pompe disease. Muscle Nerve 2012;45:319-33.

30. Morris DA; Blaschke D, Krebs A, et al. Structural and functional analyses using modern and sensitive myocardial techniques in adult Pompe disease. Int $\mathrm{J}$ Cardiovasc Imaging 2015;31:947-56.

31. Gungor D, de Vries JM, Hop WC, et al. Survival and associated factors in 268 adults with Pompe disease prior to treatment with enzyme replacement therapy. Orphanet J Rare Dis 2011;1:34.

32. Nemes A, Soliman OI, Geleijnse ML, et al. Increased aortic stiffness in glycogenosis type 2 (Pompe disease). Int J Card 2007;120:138-41.

33. Makos MM, McComb RD, Hart MN, et al. Alpha-glucosidase deficiency and basilar artery aneurysm: report of a sibship. Ann Neurol 1987;22:629-33.

34. Kretzschmar HA, Wagner H, Hubner G, et al. Aneurysms and vacuolar degeneration of cerebral arteries in late-onset acid-maltase deficiency. J Neurol Sci 1990;98:169-83.

35. Mori M, Bailey LA, Estrada J, et al. Severe cardiomyopathy as the isolated presenting feature in an adult with lateonset Pompe disease. J Inherit Metab Dis Report 2017;31:79-83.

36. Ben-Ami R, Pulgisi J, Haider T, Mehta A. The Mount Sinai Hospital clinical pathological conference: a 45 year old man with Pompe's disease and dilated cardiomyopathy. Mt Sinai J Med 2001;68:205-12.

37. Limongelli G, Elliott PM. The genetics of heart failure. Oxford textbook of heart failure. Oxford: Oxford University Press; 2011.

38. Tarnopolsky M, Katzberg H, Petrof BJ, et al. Pompe disease: diagnosis and management. Evidence-based guidelines from a Canadian expert panel. Can J Neurol Sci 2016;43:472-85.

39. Youg SP, Piraud M, Goldstein JL, et al. Assessing disease severity in Pompe disease: the roles of a urinary glucose tetrasaccharide biomarker and imaging techniques. Am J Med Genet C Semin Med Genet 2012;160C:50-8.

40. Bali DS, Goldstein JL, Banugaria S, et al. Predicting cross-reactive material (CRIM) status in Pompe disease using
GAA mutations: lessons learned from 10 years of clinical laboratory testing experience. Am J Med Genet C Semin Med Genet 2012;160C:40-9.

41. Zampieri S, Burratti E, Dominissini S, et al. Slicing mutations in glycogenstorage disease type II: evaluation of the full spectrum of mutations and their relation to patient's phenotypes. Eur J Human Genet 2011;19:422-31.

42. Van der Kraan M, Kroos MA, Joose M, et al. Deletion of exon 18 is a frequent mutation in glycogen storage disease type II. Biochem Biophys Res Commun 1994;203:1535-41.

43. Montalvo AL, Bembi B, Donnarumma $\mathrm{M}$, et al. Mutation profile of the GAA gene in 40 Italian patients with late onset glycogen storage disease type II. Hum Mutat 2006;27:999-1006.

44. Shieh JJ, Lin CY. Frequent mutation in Chinese patients with infantile type of GSD II in Taiwan: evidence for a founder effect. Hum Mutat 1998;11:306-12.

45. Becker JA, Vlach J, Raben N, et al. The African origin of the common mutation in African American patients with glycogen-storage disease type II. Am J Hum Genet 1998;62:991-4.

46. Dong-Hwan L, Wen-Juan Q, Jeongho L, et al. Hypertrophic cardiomyopathy in Pompe disease is not limited to the classic infantile - onset phenotype. J Inher Metab Dis 2014;339:71-5.

47. Prater SN, Banugaria SG, DeArmey SM, et al. The emerging phenotype of long-term surviviors with infantile Pompe disease. Genet Med 2012; 14:800-10.

48. Chen Ca, Chen YH, Hwu WL, et al. Left ventricular geometry, global function, and dyssynchrony in infants and children with Pompe cardiomyopathy undergoing enzyme replacement therapy. J Card Fail 2011;17:930-6.

49. Van der Ploeg AT, Clemens PR, Corzo $\mathrm{D}$, et al. A randomized study of alglucosidase alpha in late-onset Pompe's disease. N Engl J Med 2010;362:1396200.

50. Winchester B, Bali D, Bodamer OA, et al. Methods for a prompt and reliable laboratory diagnosis of Pompe disease: report from an international consensus meeting. Mol Gen Metab 2008;93:27581.

51. Haiking Y, Tao S, Armstrong D et al. Antibody-mediated enzyme replacement therapy targeting both lysosomal and cytoplasmic glycogen in Pompe disease. J Mol Med 2017;95:513. 